Sonderstellung der U-Gefangenen in Folge der Unschuldsvermutung insgesamt nicht gerecht. Dass Besuche „offen überwacht“ werden dürfen ( $\ 144$ Abs.1 S.1), stellt wohl nicht nur den Verfasser vor Rätsel.

Buch 2 des JVollzGB sieht in 80 Paragraphen eine umfassende Regelung des UHaftvollzuges vor, wobei weite Teile vom Strafvollzugsgesetz abgeschrieben sind. In \1 (Gestaltung des Vollzugs) erscheint die Regelung: „Den Untersuchungsgefangenen sollen Hilfen zur Verbesserung ihrer sozialen Situation angeboten werden, soweit es die besonderen Bedingungen der Untersuchungshaft zulassen." zu unverbindlich und vage. Die Zuständigkeitsregel in $\mathbb{3}$ : „...trifft die Justizvollzugsanstalt unter Beachtung der Belange des Strafverfahrens“ wird zu Streitigkeiten zwischen Anstalt und Gericht führen. Die Mindestbesuchszeit von einer Stunde im Monat ( $\mathbb{1 2}$ Abs.2) genügt den verfassungsrechtlichen Anforderungen nicht. Das Regel-Ausnahme-Verhältnis zur Überwachung von Besuchen ( $\mathbb{1 4}$ Abs.1) erscheint höchst bedenklich: „Besuche dürfen... überwacht werden, es sei denn, es liegen im Einzelfall Erkenntnisse dafür vor, dass es der Überwachung nicht bedarf.“ Umgekehrt wird verfassungsrechtlich korrekt ein Schuh daraus! Die Regelung in $\$ 20$ Abs.1: „Untersuchungsgefangenen kann gestattet werden, zu telefonieren. “ enthält keinerlei Kriterien für die Gestattung bzw. Ablehnung und ist zu restriktiv. Erfreulich konkret sind die Regelungen in $\$ 32$ zur So- zialen Hilfe. Auch, dass nach $\mathbb{3} 34$ Untersuchungsgefangenen wirtschaftlich ergiebige Arbeit angeboten werden „soll“, halte ich für richtig. Dass jedoch nach $\ 35$ Abs.1 das Arbeitsentgelt geringer ausfallen soll als das der Strafgefangenen, ist bedenklich. Insgesamt stellt der Entwurf trotz der Mängel eine brauchbare Grundlage dar.

\section{Fazit}

Positiv hervorzuheben ist, dass dem Gesetzlichkeitsprinzip durch die vorgesehenen oder bereits erlassenen Gesetze Rechnung getragen wird. In einigen Punkten werden die Haftbedingungen und die Rechtsstellung der U-Gefangenen verbessert, m.E. wird jedoch auch weiterhin eine Benachteiligung der U-Gefangenen gegenüber Strafgefangenen vorgenommen in Bereichen, in denen dies vom Haftzweck her nicht geboten ist. Die Unschuldsvermutung, das Sozialstaatsprinzip und der besondere Schutz von Ehe und Familie finden in allen Entwürfen noch zu wenig konkreten Niederschlag!

Prof. Dr. jur. Peter Höflich war zuletzt Fachhochschullehrer für Rechtswissenschaft in Cottbus, davor im Justizvollzug der Länder Nordrhein-Westfalen und Brandenburg tätig.brunnerhoeflich@gmx.de

\section{Fußnoten:}

1 z.B. NStZ 1981, 315; zutreffend die abweichende Meinung des Richters M. Hirsch, der mit Blick auf den Grundsatz des Vorbehalts des Gesetzes $\mathbb{} 119$ III StPO als Umschreibung des „,besonderen Gewaltverhältnisses“ ansieht, das seit BVerfGE 33, $1 \mathrm{ff}$. keine ausreichende Rechtsgrundlage für Grundrechtseingriffe ist

2 StV 2009, 255 ff.; NStZ 2008, 521 ff.

$3 \mathrm{StV} 2009,253 \mathrm{ff}$.

4 statt vieler: Kirschke/Brune, FS 2009, 18

$5 \mathrm{Piel} / \mathrm{Püschel/Tsambikakis/Wallau,} \mathrm{ZRP} 2009$, 33 m.w.N.

6 statt vieler: Koop, FS 2009, 6

7 Auskunft des Bayer. Staatsministeriums der Justiz vom 12.6.2009

8 Die Entwürfe bzw. das Niedersächsische Gesetz sind unter http://rsw.beck.de/rsw/upload/ ZRP/....oder unter den Internetadressen der Landtage bzw. der Landesjustizministerien zu finden

$9 \mathrm{StV} 2009,258$

10 NStZ 2008, 522

11 StV 2009, 253

12 insbes. StV 2009, 254, 256

13 insbes. StV 2009, 255

142 BvR 806/08 vom 11.6.2008 Rdnr. 30 f.

15 Paeffgen, StV 2009, 48; Piel/Püchel/Tsambikakis/Wallau, ZRP 2009, 34 m.w.N.

16 Begründung, S.95

17 Piel/Püschel/Tsambikakis/Wallau, ZRP 2009 , 34

18 BVerfG NJW 1995, $1478 \mathrm{f}$.

19 statt vieler: Kirschke/Brune, FS 2009, 19

20 BR-Drs. 829/08; vgl. Harms, FS 2009, 13 ff.

21 unveröffentlicht

22 ZRP 2009, $33 \mathrm{ff}$

23 Paeffgen, StV 2009, 46 ff.

24 Paeffgen, StV 2009, 47

25 BVerfG, StV 2008, 426

\section{Literatur:}

Forum Strafvollzug Heft 1/2009: Untersuchungshaft im Wandel?

Friedrich, K.J.: Die Normierung des Untersuchungshaftvollzuges, 2004

Koop, G./Kappenberg, B.: Untersuchungshaft - eine vergessene Reform? 1998

Paeffgen, H.-U.: Vorüberlegungen zu einer Dogmatik des Untesuchungshaftrechts, 1986

Seebode, M.: Der Vollzug der Untersuchungshaft, 1985

\title{
Europäische Impulse für die deutsche Reform der Untersuchungshaft
}

Der vorliegende Beitrag will aufzeigen, wo in der aktuellen, von den Auswirkungen der Föderalismusreform geprägten, deutschen Reformdiskussion auch europäische Einflüsse eine Rolle gespielt haben (oder hätten spielen sollen) bzw. wo weitere Impulse aus Europa zu erwarten sind. Maßgebliche europäische Akteure sind dabei der Europarat (durch die Rechtsprechung des Europäischen Gerichtshofs für Menschenrechte, der Arbeit des Anti-Folter-Komitees und durch die Europäischen Strafvollzugsgrundsätze) und die EU.

\section{Die Ausgangssituation: Die Untersuchungshaft zwischen Bund- und Länderkompetenzen}

Es ist an anderer Stelle oft und deutlich genug dargelegt worden, ${ }^{1}$ wie problematisch in Bezug auf rechtstaatliche Grundsätze die bisherige Situation der Untersuchungshaft und des Untersuchungshaftvollzugs- rechts als „Stiefkindern der Justiz“ ist. Zur noch immer aktuellen Berechtigung dieses Schlagwortes mag auch die Tatsache dienen, dass bei einer vom Greifswalder Lehrstuhl für Kriminologie durchgeführten Umfrage unter den Länderjustizministerien, welche Projekte sie in ihrem Geschäftsbereich für modellhaft im Sinne der (Fort)entwicklung eines humanen Justizvollzugs hielten, zwar insgesamt 26 Projekte aus elf Bundesländern gemeldet wurden. Nur eines jedoch befasste sich mit dem Untersuchungshaftvollzug, 
vorgestellt (und später als besonders gutes Praxismodell auch für eine Veröffentlichung ausgewählt) wurde der Wohngruppenvollzug für Untersuchungsgefangene JVA Moabit. $^{2}$

Dass in das deutsche Recht der Untersuchungshaft bzw. des Untersuchungshaftvollzuges Bewegung gekommen ist, wird vorrangig als Konsequenz der Föderalismusreform wahrgenommen: Der Bund hat die Gesetzgebungskompetenz für den Justizvollzug verloren, nach Art. 74 I GG verbleibt ihm lediglich die Kompetenz zur Regelung des "gerichtlichen Verfahrens ohne das Recht des Untersuchungshaftvollzugs“. Nach jahrzehntelanger Debatte gelingt es damit nun auf Länderebene, den Untersuchungshaftvollzug auf eine gesetzliche Grundlage zu stellen. ${ }^{3}$ Dem Bund bleibt die Aufgabe überlassen, diejenigen Normen in der StPO, die von der Kompetenzverlagerung betroffen sind - vor allem $\mathbb{\$} \$ 119 \mathrm{ff}$. StPO - neu zu fassen. ${ }^{4}$ Der Preis ist eine beklagenswerte Rechtszersplitterung ${ }^{5}$ (immerhin abgemildert durch die Tatsache, dass 12 Bundesländer von demselben Musterentwurf $(\mathrm{ME})^{6}$ ausgehen), mit schwierigen Konsequenzen insbesondere bei Kompetenzfragen, schon gar bei länderübergreifenden Sachverhalten.

Der vorliegende Beitrag konzentriert sich ergänzend zur „innerdeutschen“ Diskussion auf europäische Einflüsse auf die aktuelle Reformdebatte und auf zukünftig zu erwartende Impulse aus Europa. Auf der Ebene des Europarates werden die Rechtsprechung des Europäischen Gerichtshofs für Menschenrechte, die Arbeit des Anti-Folter-Komitees Empfehlungen des Ministerrates diskutiert. Von Seiten der EU gibt es mittelbar zwar viel Einfluss auf die Kriminalpolitik, unmittelbar wirksam geworden ist hiervon jedoch in Bezug auf die deutsche Untersuchungshaft bislang kaum etwas. Als Ausblick wird jedoch der geplante Rahmenbeschluss über die Europäische Überwachungsanordnung im Ermittlungsverfahren vorgestellt, außerdem wird von Plänen zu europäischen Mindeststandards für Anordnung und Vollzug der Untersuchungshaft seitens der EU-Kommission berichtet.

\section{Rechtsprechung des Europäischen Gerichtshofs für Menschenrechte}

Wenn es um die rechtsstaatliche Ausgestaltung des Strafverfahrens im Allgemeinen und der Untersuchungshaft im Besonderen geht, gibt zunächst das Grundgesetz den Maßstab vor. Zur Konkretisierung seiner Grundsätze bzw. der Grundrechte haben zahllose Ent- scheidungen der Obergerichte und vor allem des Bundesverfassungsgerichts beigetragen. ${ }^{7}$ Alle Gericht und Behörden sind aber auch verpflichtet, die Europäischen Menschenrechtskonvention (EMRK) und darüber hinaus auch die Judikate des Europäischen Gerichtshofs für Menschenrechte (EGMR) zu beachten. ${ }^{8}$ Zusammen dienen sie zum einen als Auslegungshilfen für Grundgesetznormen, zum anderen muss im Sinne einer „praktischen Konkordanz“ sämtliches deutsche Recht aber auch konventionsgemäß ausgelegt werden.

Wegen des starken deutsche Verfassungsrechts und der starke Stellung der deutschen Verfassungsrechtsprechung stand bislang die Straßburger Rechtsprechung trotzdem nicht im Focus der strafprozessrechtlichen Diskussion - in der Regel wurde davon ausgegangen, dass die deutschen Standards höher sind als die durch die EMRK bestimmten und durch den EGMR konkretisierten. Diese Grundannahme ist jedoch in den letzten Jahren zumindest „ein wenig erschüttert “9 worden, nachdem Deutschland mehrfach wegen Konventionsverstoßs im Zusammenhang mit Recht und Praxis der Untersuchungshaft verurteilt wurde, obwohl das Bundesverfassungsgericht zuvor nicht eingeschritten war (in zwei Fällen, in denen es um die überlange Dauer des Verfahrens gegangen war, hatte es die Fälle nicht zur Entscheidung angenommen). Die Verurteilungen durch den EGMR führten teilweise zu Änderungen der StPO.

\section{Art. 5 IV EMRK: Justizförmiges Verfahren bei der Haftkontrolle}

Art. 5 IV EMRK sieht eine richterliche Haftkontrolle vor, in der Zusammenschau mit Art. 5 III und Art. 6 (Recht auf ein faires Verfahren) EMRK führt dies zu einem umfassenden Rechtsschutz, der das Gebot der Waffengleichheit achtet (d. h. auch einen Anspruch auf rechtlichen Beistand einschließt) und unter anderem voraussetzt, dass der Beschuldigte ausreichende Informationsmöglichkeiten hat, um sich gegen den Haftbefehl bzw. die Anordnung der Haftfortdauer zur Wehr zu setzen. Vor diesem Hintergrund war in Deutschland schon lange $\mathbb{} 147$ II StPO kritisiert worden, der bei Gefährdung des Untersuchungszwecks die Akteneinsicht des Verteidigers vor Abschluss der Ermittlungen einschränkt. In dem vom EGMR zuletzt behandelten Fall ${ }^{10}$ hatte der Verteidiger, der für den Beschuldigten eine Haftprüfungsantrag gestellt hatte, keine Akteneinsicht erhalten, sondern nur von der Staats- anwaltschaft mündliche Informationen aus dem Akteninhalt angeboten bekommen. In der Entscheidung wurde unter Berufung auf frühere Entscheidungen ${ }^{11}$ einstimmig ein Verstoß gegen Art. 5 IV EMRK festgestellt. Zur Begründung wurde neben der Tatsache, dass das Haftprüfungsverfahren zu langsam geführt worden war, angeführt, dass die Verweigerung des Zugangs zu Dokumenten, die zur Überprüfung der Rechtmäßigkeit der Inhaftierung „essentiell“ waren, gegen das Gebot der Waffengleichheit und damit gegen Art. 5 IV verstoße.

Diese Rechtsprechung nahm die Bundesregierung zu Anlass, $\$ 147$ II StPO zu überarbeiten. Im ursprünglichen Referentenentwurf war nur davon die Rede, dass „dem Verteidiger die für die Beurteilung der Rechtmäßigkeit der Freiheitsentziehung wesentlichen Informationen in geeigneter Weise zugänglich zu machen sind ". Nachdem viele Experten und auch Parlamentarier (insbesondere im Rechtsausschuss) erhebliche Zweifel geäußert hatten, ${ }^{12}$ dass diese Formulierung die Rechtsprechung des EGMR tatsächlich umsetzt, wurde der Entwurf um den Zusatz ergänzt, „in der Regel ist insoweit Akteneinsichtsrecht zu gewähren “.

Im Verlauf der Beratungen kam es dann zu einer Überraschung: Das reformierte Untersuchungshaftrecht wird einen neuen $\$ 141$ III StPO enthalten, der mit Inkrafttreten am 1.1.2010 die notwendige Verteidigung in allen Fällen vorsieht, in denen Untersuchungshaft oder eine einstweilige Unterbringung tatsächlich vollstreckt wird. Was war geschehen? Bereits in der ersten Beratung des Gesetzentwurfs, verstärkt durch die entsprechenden Stellungnahmen der Experten, zeichnete sich ab, dass die Mitglieder der Rechtsausschusses in seltener Einmütigkeit eine Verteidigung in U-Haft-Fällen „von Anfang an“ befürworteten, unter anderem mit dem Argument der Benachteiligung armer bzw. sprachunkundiger oder anderweitig benachteiligter Beschuldigter, die regelmäßig die ersten drei Monate in U-Haft unverteidigt verbrächten. Diese Forderung ist von Wissenschaftlern mit dem Argument, dass sich so beachtliche Verkürzungen der Untersuchungshaft erreichen lassen, seit Jahrzehnten erhoben worden. ${ }^{13}$ Insofern verwundert die Weichenstellung in letzter Minute. Sie ist möglicherweise dem Umstand geschuldet, dass die beteiligten Parlamentarier überwiegend selbst Strafrechtspraktiker, in der Regel Strafverteidiger sind. Möglicherweise hat auch der stete Tropfen den Stein gehöhlt - die Mehrkosten 
der Pflichtverteidigung können unter Umständen durch die Verkürzung der U-HaftZeiten ausgeglichen werden, auch dieses Argument fand endlich Gehör. Ein weiterer Umstand spielte aber mittelbar auch eine Rolle: Der EGMR fordert Waffengleichheit für den Beschuldigten - Akteneinsicht kann aber nach deutschem Verständnis nur sein Verteidiger haben. Versteht man also die Entscheidungen des EGMR in der oben dargestellten Weise, dass nämlich vernünftige Verteidigungsaussichten in der Regel nur nach Kenntnis der wichtigen Aktenteile möglich sind, ergibt sich die frühe notwendige Verteidigung auch hieraus.

Die wiederholten Verurteilungen Deutschlands durch den EGMR haben also unmittelbar zu einer Änderung des $\ 147$ II StPO geführt und mittelbar der Forderung nach notwendiger Verteidigung bei vollzogener U-Haft Schützenhilfe gegeben.

\section{Art. 5 III und Art. 6 I EMRK: Das Beschleunigungsgebot in Haftsachen}

Der EGMR hatte darüber hinaus in mehreren Fällen wegen überlanger Dauer des Verfahrens (und damit einhergehend der Untersuchungshaft) in Verfahren gegen Deutschland eine Verletzung von Art. 5 EMRK zu untersuchen, Deutschland wurde dabei mehrfach verurteilt. ${ }^{14}$ Die rechtstatsächlichen Befunde sind dabei gemischt: Die Stichtagszahlen bei den Untersuchungsgefangenen gehen zurück, die Länge der U-Haft hingegen nimmt für die Betroffenen aber tendenziell zu: Der Anteil derjenigen Verurteilten, der sechs Monate und länger in Untersuchungshaft verbringt, betrug 2006 fast ein Viertel; 1990 waren es weniger als $18 \% .{ }^{15}$ Betrachtet man deliktsspezifisch Daten für 2003-2006 (Westdeutschland) ist für alle Delikte (ohne Verkehrsstraftaten) eine geringfügig zunehmende Länge festzustellen, besonders deutlich fällt die Zunahme tatsächlich aber bei Delikten gegen das Leben aus, wo vor allem Haftzeiten von über einem Jahr deutlich zugelegt haben. ${ }^{16}$

Auch hier wurde versucht, den Rückenwind aus Straßburg zu nutzen, um auf eine feste Höchstgrenze der Untersuchungshaft zu drängen ${ }^{17}$ - ebenfalls eine sehr alte Forderung. ${ }^{18}$ In diesem Zusammenhang ist allerdings anzumerken, dass der EGMR sich selbst schwer tut, zeitlich starre Grenzen für die Höchstdauer der Untersuchungshaft zu setzen: So urteilte der Gerichtshof, dass im letzten der (in der Sache) entschiedenen deutschen Fälle ${ }^{19}$ keine Verletzung von Art. 5 III EMRK vorlag, obwohl der Beschwerde- führer mehr als fünf Jahre und sechs Monate in deutscher U-Haft saß (vor seiner Auslieferung war er bereits im Libanon inhaftiert). Der Gerichtshof argumentierte, dass die exorbitante Länge der U-Haft - es handelte sich um das La-Belle-Verfahren in Berlin - durch die besonders schwierigen und umfangreichen Ermittlungen bzw. Zeugenvernehmungen in den Hauptverhandlungen im konkreten Fall noch gerechtfertigt war.

In einer umfangreichen rechtsvergleichenden Studie der Universitäten Tilburg (Niederlande) und Greifswald zur Untersuchungshaft in Europa ${ }^{20}$ wurde zudem festgestellt, dass Aussagen über den Nutzen von festen Zeitlimits schwierig sind. ${ }^{21}$ Zum einen wird schon der Begriff der Untersuchungshaft unterschiedlich verstanden - manche europäischen Staaten gebrauchen den Terminus nur für die Zeit bis zum ersten Sachurteil, die Mehrheit verwendet ihn für die Haft bis zum rechtskräftigen Urteil. Naturgemäß ist der Zeitraum, über den sich Untersuchungshaft erstrecken kann, dann sehr unterschiedlich. Außerdem kann es geschehen, dass Fristen (die, falls sie existieren, oft sehr lang sind) von den Justizbehörden ausgeschöpft werden, wohingegen Staaten, die zeitliche Begrenzungen im konkreten Fall allein aus dem Verhältnismäßigkeitsgrundsatz ableiten und/oder kurze Haftprüfungsfristen haben, relativ kürzere tatsächliche Haftzeiten haben (wobei die komparative Messung von Haftzeiten derzeit unmöglich ist - selbst wenn nationale Daten vorliegen, sind sie wegen unterschiedlicher Konzepte und Zählweisen nicht miteinander zu vergleichen). Insofern gab es im Bereich der zeitlichen Begrenzung von Untersuchungshaft keinen europäischen Niederschlag im deutschen Haftrecht.

\section{Die Berichte des Anti-Folter- Kommitees (CPT)}

Nach Angaben der Entwurfsbegründung flossen in den Entwurf für die neuen bundesgesetzlichen Regelungen auch Erkenntnisse aus Berichten des CPT (Besuche im November 2005 und Dezember 2000) ${ }^{22}$ ein, die sich in klareren Bestimmungen zu den Belehrungspflichten sofort nach der Festnahme beziehen ( $\mathbb{S} 114 \mathrm{a}$-e StPO-E). Dabei ging es insbesondere um das Recht auf die Untersuchung durch einen Arzt nach Wahl des Beschuldigten; außerdem um das Recht zur Benachrichtigung von Angehörigen oder nahestehenden Personen unmittelbar nach der Festnahme (d. h. nicht erst nach der förmlichen „Verhaftung“). Außerdem gab es im Rahmen dieser Klarstellungen in Bezug auf die Fürsorgepflicht für sprachunkundige Festgenommene. Diese Bestimmungen setzen das um, was durch das CPT verlangt worden ist, inwiefern sie praktisch durchgesetzt werden können, bleibt abzuwarten. ${ }^{23}$

Mehr Grund als der Bund mit seiner Restkompetenz haben jedoch die Länder bei ihren Entwürfen für Untersuchungshaftvollzugsgesetze auf die CPT-Berichte zu achten. Zwar hat das CPT in den letzten Jahren Untersuchungshaftanstalten nur mit Blick auf die Situation von Abschiebehäftlingen besucht, gleichwohl geben Beobachtungen sowohl in den Deutschlandberichten als auch in den inzwischen etablierten CPT-Standards, ${ }^{24}$ die als Quintessenz der Erkenntnisse aus den Ländervisiten der vergangenen 20 Jahre entstanden sind, Anlass für Veränderungen beim Vollzug der Untersuchungshaft.

Im Mittelpunkt stehen dabei Forderungen des CPT an alle besuchten Staaten nach „meaningful activities“ während der Untersuchungshaft. Defizite wurden diesbezüglich in nahezu jedem Bericht über besuchte U-Haft-Anstalten geltend gemacht. In den Berichten findet sich stets eine Formulierung, die in etwa lautet: „Die Untersuchungsgefangenen verbringen 23 Stunden am Tag sich selbst überlassen in ihrer Zelle." Die deutschen Anstalten entsprechen wohl dem europäischen Niveau in dieser Hinsicht - und das ist ein bedenklicher Befund. Insofern ist grundsätzlich jede Normierung von Angebotsstrukturen durch die Untersuchungshaftvollzugsgesetze zu begrüßen, wenngleich manche Formulierungen schwach sind oder deutlich hinter den Standards zurückbleiben.

So ist z. B. der gemeinsame Musterentwurf im Hinblick auf den Hofgang kein Fortschritt: Der Außenaufenthalt zählt zwar traditionell zu den Gesundheitsfürsorgemaßnahmen, da er aber - wie oben angedeutet - gelegentlich außer den Mahlzeiten das einzige ist, was den Tagesablauf strukturiert, bekommt er faktisch schon den Charakter einer „Beschäftigung “. Insofern wäre es besser, auch in der Formulierung herauszustellen, dass $\mathbb{2} 20$ Abs. 2 ME nur das absolut und unter allen Umständen zu gewährende Minimum darstellt, in etwa so: „Der Aufenthalt im Freien wird den Untersuchungsgefangenen täglich ermöglicht. Er darf eine Stunde in keinem Falle unterschreiten." Die Aufnahme des $\$ 24$ Abs. 2 ME mit einem regelmäßigen Arbeitsangebot für U-Gefangene stellt hingegen eine echte Errungenschaft des Musterentwurfs ${ }^{25}$ gegenüber der bis- 
herigen Praxis und der damit verbundenen Schlechterstellung der U-Gefangenen gegenüber den Strafgefangenen dar. Die Justizverwaltungen werden sich daran messen lassen müssen, wie es ihnen gelingt, tatsächlich sinnvolle Arbeit zur Verfügung zu stellen. Im Hinblick auf die Freizeitangebote $(\mathbb{\$} 26$ ME) ist die Vorgabe („Zur Freizeitgestaltung sind geeignete Angebote vorzuhalten. Insbesondere sollen Sportmöglichkeiten und Gemeinschaftsveranstaltungen angeboten werden. “) allerdings etwas dünn, zum Regelangebot sollte z. B. auch der Zugang zu einer vernünftig bestückten Anstaltsbibliothek mit diversen Medien gehören. Auf Probleme im Hinblick auf Außenkontakte soll gleich unten bei der Erörterung der Vorgaben der Europäischen Strafvollzugsgrundsätze noch eingegangen werden.

Weiterhin hat das CPT anlässlich des Besuchs 2005 in einer sog. „unmittelbaren Beobachtung", die gesondert und unverzüglich noch vor Fertigstellung des Berichts der deutschen Regierung mitgeteilt wurde, ${ }^{26}$ ausdrücklich Bezug auf bestimmte Praktiken bei der Fesselung und auch Fixierung von Gefangenen genommen. Die Praxis, die in allen besuchten Anstaltstypen angetroffen wurde, ${ }^{27}$ wurde von den Delegationsmitgliedern des CPT als besorgniserregend empfunden. Sie machten konkrete Vorgaben, wie sie zukünftig auszugestalten sei. Wörtlich hieß es im Bericht (deutsche Übersetzung, S. 10): “... und forderte alle zuständigen Behörden auf, dafür zu sorgen, dass der Zustand jeder fixierten Person unverzüglich von einem Arzt überprüft wird, dass die betreffende Person nicht mit Polizeihandschellen in ihrer Bewegungsfreiheit beschränkt wird und dass sie von einem Mitarbeiter ständig, unmittelbar und persönlich überwacht wird (Sitzwache)." Diese Vorgaben sind in den Vorschriften zur Fesselung/Fixierung ( $\$ 49$ II Nr. 5 und 6; $\mathbb{5} 51 \mathrm{ME}$ ) nicht vollständig umgesetzt - zwar schreibt $\$ 53 \mathrm{ME}$ alsbaldige und regelmäßige Überprüfung durch einen Arzt vor, geht jedoch nicht über $\mathrm{Nr}$. 65 UVollzO hinaus. Insbesondere der letzte Punkt (Überwachung durch Mitarbeiter, ggf. Sitzwache) der CPT-Beobachtung fehlt in den Gesetzentwürfen.

\section{Die Empfehlungen des Europarates}

Als weitere Konkretisierung der EMRK, jedoch ohne verbindlichen völkerrechtlichen Status, gibt es einige Empfehlungen ${ }^{28}$ des Europarates, die im Bereich der Untersuchungshaft wirksam werden. In Bezug auf die Charakterisierung von europäischen oder internationalen Standards als bloßes „soft law" ist aus deutscher Sicht allerding seit der Jugendstrafvollzugsentscheidung des Bundesverfassungsgerichts stets zu betonen, dass es ,auf eine den grundrechtlichen Anforderungen nicht genügende Berücksichtigung vorhandener Erkenntnisse oder auf eine den grundrechtlichen Anforderungen nicht entsprechende Gewichtung der Belange der Inhaftierten“ hindeuten kann, ,wenn völkerrechtliche Vorgaben oder internationale Standards mit Menschenrechtsbezug, wie sie in den im Rahmen der Vereinten Nationen oder von Organen des Europarates beschlossenen einschlägigen Richtlinien und Empfehlungen enthalten sind ..., nicht beachtet beziehungsweise unterschritten werden. “29

\section{Die Empfehlung (2006) 13 zur Untersuchungshaftpraxis: Deutsche Defizite im Hinblick auf gesetzliche Ausnahmen bei der U-Haftanrechnung und bei der freien Arztwahl}

Zunächst ist hier die Recommendation (2006) 13 on the "Use of remand in custody, the conditions in which it takes place and the provision of safeguards against abuse" zu nennen, die „Empfehlung des Ministerrates über den Gebrauch der Untersuchungshaft, den Bedingungen, unter denen sie vollzogen wird, und die Vorkehrungen gegen ihren Missbrauch". Obwohl es, wie oben angedeutet, eine Vielzahl von Entscheidungen des Gerichtshofes zur Untersuchungshaft, gibt, bestand nach Auffassung des Europarates ein Bedarf an einem konkreteren Instrument, unter anderem deshalb, weil das Wesen des Verfahrens vor dem EGMR (Art. 32-34, 46 EMRK) Entscheidungen nur inter partes Wirkung verleiht, so dass aus dem case law nur eingeschränkt und zögernd tatsächliche gemeinsamen europäische Standards entstehen. Es wurde daher versucht, die Kernaussagen der ständigen Rechtsprechung des EGMR zur Untersuchungshaft in die Form eines einheitlichen Instrumentes zu gießen. Der Europarat betont in den Erwägungsgründen, wie wichtig ,internationale Normen“ mit Blick auf die Rechtfertigung von Untersuchungshaft, auf das Verfahren ihrer Anordnung und Aufrechterhaltung sowie im Hinblick auf die Haftbedingungen, seien - hinzu kommen müsse aber auch eine effektive Implementierung. Betrachtet man die Vorrede, so hat auch der Europarat sicher eine gewisse Harmonisierung des europäischen Rechts im Blick, die vor allem für grenzüberschreitende Sachverhalte wichtig wird. In der Präambel der Empfehlung
(2006) 13 ist die Intention des Instruments umschrieben: So sollen strikte Grenzen für den Gebrauch der Untersuchungshaft gesetzt und Alternativen so weit als möglich gefördert und richterliche Beschlüsse zur Anordnung und Fortdauer von Untersuchungshaft, aber auch für ihre Alternativen, festgeschrieben werden. Ferner müssen danach Untersuchungshaftbedingungen, die „dem rechtlichen Status, der auf der Unschuldsvermutung basiert, angemessen sind" geschaffen werden. Schließlich sollen effektive Schutzmechanismen etabliert werden, wenn die in der Empfehlung aufgestellten Standards verletzt werden.

Es liegt nahe, bei diesem Versuch die Einzelfall-Rechtsprechung des EMRK wieder zu aggregieren, anzunehmen, dass sich wenig neue Impulse für die deutsche Diskussion finden. Tatsächlich gehen sie jedoch zumindest theoretisch in einigen Punkten hierüber hinaus: Regel 6 schreibt fest, dass Untersuchungshaft generell nur für Beschuldigte verfügbar sein soll, die Straftaten begangen haben sollen, die mit Freiheitsstrafe „bedroht“ („imprisonable“) sind. Auch wenn \113 StPO Einschränkungen bei Bagatelltaten vorsieht, ist der Erlass von Untersuchungshaft nach dem Wortlaut der Norm auch dann nicht ausgeschlossen, wenn nur eine Geldstrafe zu erwarten ist, ${ }^{30}$ nicht einmal dann, wenn, wie teilweise im Nebenstrafrecht, grundsätzlich nur eine Geldstrafe gesetzlich angedroht ist. Hier ist jedoch stets ein Verstoß gegen den Verhältnismäßigkeitsgrundsatz anzunehmen. ${ }^{31}$ Außerdem ist zuzugeben, dass die Einschränkung „mit Freiheitsstrafe bedroht“ in aller Regel kein echter Gewinn für die Verhältnismäßigkeitsprüfung ist: In Deutschland wie in den meisten europäischen Staaten ist selbst für Bagatelltaten abstrakt eine Strafandrohung, die Freiheitsstrafe einschließt und zumeist auch über einem Jahr liegt, vorgesehen. ${ }^{32}$ Die praktische Bedeutung dieser Regel Nr. 6 ist daher gering.

Auch in Bezug auf die Länge der Untersuchungshaft hängt die Empfehlung in Nr. 22 (2) in ihrer Muss-Vorschrift lediglich eine relativ niedrige Messlatte auf: Danach darf die Untersuchungshaft nicht über die abstrakt angedrohte Freiheitsstrafe (,the penalty that may be imposed“) hinausgehen, „normalerweise“ darf sie auch nicht „außer Verhältnis“ zu ihr stehen („,nor normally be disproportionate to “). In Deutschland wird überwiegend davon ausgegangen, dass die Untersuchungshaft in ihrer Wirkung nicht der Freiheitsstrafe gleichkommen dürfe 
- daraus folgt zumindest, dass sie ihre konkret erwartete Länge nicht überschreiten darf. Weitergehend wird der Zwei-DrittelZeitpunkt der erwartbaren Freiheitsstrafe als Höchstgrenze betrachtet, dem folgt teilweise auch die Rechtsprechung. ${ }^{33}$ Kritiker haben darüber hinaus stets (tw. erheblich) weitergehende Vorschläge zur zeitlichen Begrenzung gemacht (siehe oben). Auch hier bringt die Empfehlung (2006) 13 daher keine greifbaren Impulse.

Im Hinblick auf das deutsche Recht relevant ist hingegen die nach Nr. 33 der Empfehlung 2006 (13) zwingende Regelung, dass die in Untersuchungshaft verbachte Zeit von der verhängten Freiheitsstrafe abgezogen wird - $\int 51$ I S. 2 StGB ist (auch) vor diesem Hintergrund nicht haltbar, ebenso wie die Bestimmung des $\$ 52$ a I S. 2 JGG. Beide Vorschriften sind allerdings nicht Gegenstand der aktuellen Reformdebatte geworden.

Relativ konkret wird die Empfehlung (2006) 13 auch in bezug auf die „Fortführung medizinischer Behandlung “ in Nr. 37. Nach Nr. 37 II müssen Untersuchungsgefangene grundsätzlich das Recht haben, einen Arzt nach ihrer Wahl zu konsultieren und sich auch von ihm (weiter) behandeln zu lassen. Auch hier spricht die Unschuldsvermutung für eine grundsätzlich freie Arztwahl. Die

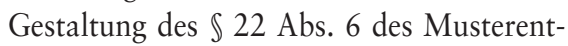
wurfes für ein UntersuchungshaftvollzugG (anders als z. B. im Entwurf aus BadenWürttemberg) als „Soll-Regelung“ ist daher grundsätzlich zu begrüßen. Es muss aber noch klargestellt werden, dass es eben nicht nur um einen „Rat“, sondern - wie in $\mathrm{Nr}$. 37 II klargestellt - auch um eine Behandlung geht. Problematisch ist die Regelung des Musterentwurfs, wonach die Erlaubnis, einen Wahlarzt zu konsultieren, von einer Schweigepflichtsentbindung abhängig gemacht werden kann (immerhin ist sie aber nicht zwingend).

\section{Die Europäischen}

Strafvollzugsgrundsätze: Deutsche Probleme vor allem im Hinblick auf die Außenkontakte

In Bezug auf andere Modalitäten des Untersuchungshaftvollzugs verweist die Empfehlung (2006) 13 auf die spezielleren Europäischen Strafvollzugsgrundsätze. ${ }^{34}$ Auch sie haben lediglich Empfehlungscharakter und stellen kein bindendes Völkerrecht dar, jedoch ist auch für sie die oben angesprochene Auffassung des Bundesverfassungsgericht zu beachten, außerdem haben sie anders als viele andere Empfehlungen des Europarates innerhalb von Europa und darüber hinaus beachtliche Verbreitung und Anerkennung gefunden. Ihr siebter Teil ist dem Vollzug der Untersuchungshaft gewidmet. Im vorliegenden Beitrag sollen nur einzelne Beispiele für ihre Umsetzung bzw. Umsetzungsdefizite bei den Untersuchungshaftvollzugsgesetzentwürfen aufgezeigt werden.

In diesem Zusammenhang ist allgemein ist zu sagen, dass der Musterentwurf und die entsprechenden Länderentwürfe einige konstruktive Neuerungen mit sich bringen, die zu begrüßen sind - auf das Arbeitsplatzangebot ist schon eingegangen worden, Regelungen wie die zur Suizidprophylaxe im Brandenburgischen Entwurf ( $\$ 5$ BrbUVollzG-E) oder die zur sozialen Hilfe und Vernetzung mit Akteuren von außen $(\mathbb{S} 6$ $\mathrm{ME}$ ); erzieherische Ausgestaltung des Vollzugs der Untersuchungshaft an jungen Gefangenen durch Bereitstellung von Schul-, Ausbildungs- und Freizeitangebote, Erhöhung der Besuchsfrequenz bzw. -dauer etc. (11. Abschnitt bzw. $\$ \int 66 \mathrm{ff}$. ME) sind auch hierzu zu zählen. Es muss aber betont werden, dass die Regelungen nur dann zu einer echten Verbesserung der Situation führen werden, wenn sie organisatorisch, personell und finanziell untersetzt sind. In den Begründungen der Entwürfe wird auch darauf hingewiesen, dass finanzielle Mehraufwendungen nicht zu vermeiden sind. Nicht alle Entwürfe bekennen jedoch deutlich Farbe, was Anlass zur Besorgnis im Hinblick auf die praktische Umsetzung der Vorgaben gibt.

So ist einerseits der Berliner Entwurf (Punkt III der Entwurfsbegründung, „Kosten“) deutlich, wenn er finanzielle Mehraufwendungen als unumgänglich bezeichnet - sowohl für Personal- als auch für Sachkosten. Problematischer ist die Begründung des UVollzG-E von Mecklenburg-Vorpommern vom 6.5.2009, hier heißt es in der Begründung zwar „Mehraufwendungen seien unumgänglich“, sie seien jedoch „überschaubar" - in der Spezifizierung ist z. B. nicht von höheren Personalkosten die Rede. Auch die Einleitung der Drucksache mit dem brandenburgischen Entwurf (Drs. 4/7334 des brandenburg. Landtags) verwundert: Hier heißt es wörtlich unter der Überschrift „Zweckmäßigkeit“. „Der Untersuchungshaftvollzug war bisher gesetzlich nicht geregelt. Verglichen mit dem Strafvollzugsgesetz des Bundes handelt es sich eine „schlankere“ Regelung." Mal abgesehen davon, dass die Bedeutung dieser Formulierung in der Sache nicht ganz klar ist, muss der Auffassung ent- gegengetreten werden, eine Verbesserung der Situation sei „schlank“ oder „kostenneutral“" zu haben. Es ist daher auch irreführend, wenn an derselben Stelle (A.III) zwar davon die Rede ist, dass „Mehrkosten (Sachkosten) unumgänglich“ seien, aber „Diese führen aufgrund der geringen Belegungsdichte im Untersuchungshaftvollzug des Landes Brandenburg jedoch im Ergebnis nicht zu einer Erhöhung der Ausgaben." Das mag derzeit richtig sein und die geringe Belegungsdichte ist überaus erfreulich, aber die Situation kann sich relativ schnell ändern. Da die Regelungen im Gesetzentwurf jedoch rechtstaatlichen Vorgaben (Sozialstaatsprinzip! Unschuldsvermutung!) folgen, sind sie entsprechend strukturell abzusichern. Sich hierbei (dann) auf fehlende öffentliche Mittel zu berufen, würde im Übrigen nicht nur gegen Verfassungsrecht, ${ }^{35}$ sondern auch gegen $\mathrm{Nr}$. 4 der Europäische Strafvollzugsgrundsätze verstoßen, der festhält: „Mittelknappheit kann keine Rechtfertigung sein für Vollzugsbedingungen, die gegen die Menschenrechte von Gefangenen verstoßen. “

Wesentlich konkreter ist der folgende Punkt: Es erscheint in Deutschland offenbar fernliegend, konkrete gesetzliche Bestimmungen zur Hygiene im Gesetz festzuschreiben. Exemplarisch geht es um das Recht auf regelmäßiges Duschen - es fehlt in allen Gesetzentwürfen. Dass sich im Strafvollzugsgesetz hierzu auch keine Regelung findet, ist kein Argument - es soll ja hier um eine Angleichung der Bedingungen $(\mathbb{S} \mathrm{ME})$ in Freiheit für einen als unschuldig geltenden Bürger gehen. Die Europäischen Strafvollzugsgrundsätze sehen in Nr. 19.4 vor, dass alle Gefangene möglichst täglich, wenigstens aber zwei Mal pro Woche, die Gelegenheit zum Duschen haben müssen. Soweit ersichtlich, ist dieser Standard bei weitem nicht in allen deutschen Anstalten eingehalten und Defizite tragen zur Belastung während der Untersuchungshaftzeit durchaus bei. Es wäre daher sinnvoll, eine entsprechende Vorschrift in die Gesetze aufzunehmen.

Ein weiterer, überaus kritischer Punkt, bei dem europäischen Standards nicht bzw. nicht ausreichend umgesetzt worden sind, sind die Kontakte zur Außenwelt: Sie sind, wie auch die Europäischen Strafvollzugsgrundsätze in $\mathrm{Nr}$. 24.1 betonen, unerlässlich für Aufrechterhaltung von Verbindungen in das soziale Leben und haben Auswirkungen auf das Anstaltsklima hat. Hier ist neben der Unschuldsvermutung bei Untersuchungsgefangenen ein weiterer grundrechtsrelevanter Aspekt zu berücksichtigen: Bei 
nahen Angehörigen ist das Recht aus Art. 6 GG betroffen, was jüngst auch vom Bundesverfassungsgericht ${ }^{36}$ betont wurde. Unzureichende Kontaktmöglichkeiten verletzen daher mehrere Grundrechtsträger. Grundsätzlich normieren hier die Europäischen Strafvollzugsgrundsätze in $\mathrm{Nr}$. 99, dass immer dann, wenn nicht ein im Einzelfall begründetes Verbot einer Justizbehörde vorliegt, Untersuchungsgefangene zusätzliche (im Vergleich zu Strafgefangenen) Kommunikationsmöglichkeiten haben müssen.

Dem tragen die Untersuchungshaftvollzugsgesetzentwürfe nicht ausreichend Rechnung: So verstößt $\mathbb{S} 40 \mathrm{ME}$ in der jetzigen Formulierung, die den Untersuchungsgefangenen „gestatten kann, auf eigen Kosten Telefongespräche zu führen“, gegen die oben genannten Regelungen: Die Gestattung von Telefonaten muss der Regelfall sein, so dass das Gesetz an dieser Stelle nur Einschränkungen nennen sollte. Soweit technische, bauliche oder andere Gründe für die Gestaltung der Norm ausschlaggebend sein sollten, ist zu fordern, dass auch Justizvollzugsanstalten von den bemerkenswerten modernen technischen Möglichkeiten Gebrauch machen müssen, um entsprechende Telefonanlagen (z. B. auch mit Freischaltung einzelner Nummern o. ä.) zu installieren. Ähnliches gilt für den Paktempfang ( $\$ 41$ $\mathrm{ME})$ : Auch hier müsste an sich die grundsätzliche Zulässigkeit der Ausgangspunkt sein und die Vorschrift Einschränkungen normieren. Allerdings ist zuzugeben, dass wegen des aus Sicherheitsgründen (in Bezug auf die Anstalt) erforderlichen Kontrollaufwandes nicht dieselben Maßstäbe anzulegen sind wie bei Telefonaten, so dass eine Beschränkung auf eine bestimmte Zahl von Paketen vertretbar erscheint. Die grundsätzliche Unzulässigkeit des Empfangs von Nahrungsmittelpaketen, die in einigen UVollzG-E vorgesehen ist (z. B. Musterentwurf, Berlin, Mecklenburg-Vorpommern, Hamburg ( $\$ 28$ HambUVollzG-E, gilt nicht für Jugendliche); Brandenburg dagegen schließt den Empfang von Nahrungsmittelpaketen nicht grundsätzlich aus), verstößt jedoch gegen die Europäischen Strafvollzugsgrundsätze.

Nach Nr. 99 (a) der Europäischen Strafvollzugsgrundsätze müssen Untersuchungsgefangene mehr Besuchsmöglichkeiten haben als Strafgefangene, für letztere bestimmt Nr. 24.1, dass „so oft wie möglich“ Besuch möglich sein soll. Das ist natürlich wenig konkret. Die Minimalforderung, dass nämlich Untersuchungsgefangene mehr Besuch haben dürfen als Strafgefangene wird zumindest von Musterentwurf aufgegriffen: die Besuchszeiten wurden gegenüber der allgemeinen Regelung auf 2 Stunden im Monat erhöht. Gesetzentwürfe, die selbst dahinter zurückbleiben, verstoßen ganz klar gegen die Europäischen Strafvollzugsgrundsätze. Aber auch diese Minimalregelung greift zu kurz: Eine Mindestbesuchszeit von 4 Stunden pro Woche auch für die Erwachsenen (nach $\$ 72$ des Musterentwurfes sind für junge Untersuchungsgefangene 4 Stunden vorgesehen) zu fordern, ebenso, dass Besuche von Kindern (Art. 6 GG!) nicht auf die Regelbesuchszeit anzurechnen sind - hier ist in der Grundrechtsbetroffenheit der kindlichen Besucher kein Unterschied zu machen. Das ist weiter damit zu begründen, dass die wöchentliche Frequenz zur Aufrechterhaltung sinnvoller Familienkontakte gerade in der schwierigen und ungewissen U-Haft-zeit wichtig ist, zum anderen die typischen Halb-Stunden-Besuche für die Beteiligten (Drittwirkung auf die Angehörigen!) oft zu kurz sind, um „sinnvoll“ genannt zu werden. Erfahrungsgemäß wird außerdem die im Gesetz angegebene Mindestbesuchszeit zur Regelbesuchszeit, so ist es auch - soweit ersichtlich - z. B. trotz allerorts sinkender Belegungszahlen nicht zu einer Ausweitung der Besuchszeiten bzw. -frequenzen gekommen, obwohl hierfür nun ja an sich mehr personelle Kapazitäten zur Verfügung stehen müssten.

Vorzugswürdig wäre eine Regelung, nach der „so häufig wie möglich, mindestens aber vier Stunden monatlich" Besuch empfangen werden darf. Das würde die Justizverwaltung zwingen, in diesem eminent wichtigen Punkt elastisch auf freiwerdende Kapazitäten zu reagieren. Dass hier nicht vollkommen abwegige Forderungen gestellt werden, mag ein Blick ins Ausland verdeutlichen: In Portugal und Belgien beispielweise kann nach dem Gesetz Besuch grundsätzlich jeden Tag empfangen werden, in Österreich und Bulgarien mindestens zweimal wöchentlich. ${ }^{37}$ In England und Wales können unverurteilte Gefangene in der Regel drei Mal pro Woche für eine Stunde Besuch empfangen, hier sind, nebenbei bemerkt, sogar finanzielle Beihilfen zur Ermöglichung des Besuchs im Rahmen des Assisted Prison Visits Scheme möglich. ${ }^{38}$

\section{Die Europäischen Grundsätze zum Haftvollzug/Strafvollstreckung bei Jugendlichen: Umsetzung des Trennungsgrundsatzes?}

Im Hinblick auf die Europaratsinitiativen soll abschließend noch darauf hingewiesen werden, dass sowohl die Europäischen Strafvollzugsgrundsätze als auch die spezielleren Empfehlungen des Europarats für Jugendliche im Vollzug ambulanter und freiheitsentziehender Sanktionen von 2008 ("European Rules for Juvenile Offenders Subject to Sanctions and Measures (ER JOSSM)“, Rec (2008) 11) Vorgaben für die Verhängung (bzw. Vermeidung) von Untersuchungshaft bei Jugendlichen und in den Nr. 108-113 deren Vollzug machen. ${ }^{39}$ Hier soll aus Platzgründen nur auf den Trennungsgrundsatz eingegangen werden: Die Europäischen Empfehlungen formulieren den doppelten Trennungsgrundsatz (Untersuchungs- von Strafgefangenen und jugendliche von erwachsenen Gefangenen) als Muss-Vorschrift (Nr. 59.1 ERJOSSM. Im deutschen Musterentwurf $(\mathbb{S} 11(2))$ ist dieses Trennungsgebot weniger strikt gefasst, so kann etwa aus Gründen der „Sicherheit und Ordnung der Anstalt" von getrennter Unterbringung abgewichen werden. Gleichwohl stellt dies im Hinblick auf die europäischen Standards gegenüber der UVollzO eine erhebliche Verbesserung dar, da klargestellt wird, dass keinesfalls von einer Vollzugsgestaltung nach $\$ 67$ - d. h. nach erzieherischen Gesichtspunkten und unter Bereitstellung von altersgemäßen Bildungs- und sonstigen Beschäftigungsangeboten - abgewichen werden darf.

\section{Initiativen der Europäischen Union}

Als Ausblick soll abschließend noch auf Initiativen der Europäischen Union im Hinblick auf die Untersuchungshaft eingegangen werden. 2004 sollten mit dem Haager Programm $^{40}$ Konturen einer „kohärenteren“ Strafverfolgungs- und Kriminalpolitik der EU entwickelt werden, vorangegangen war das Programm von Tampere, inzwischen gibt es einen Stockholmer Nachfolger. ${ }^{41}$ Das Programm wurde begleitet von einem „Action Plan“, der - entsprechend Art. 29 EU-Vertrag - dazu beitragen soll, dass „die Bürger mit einem hohen Sicherheitsniveau in einem gemeinsamen Raum der Freiheit, der Sicherheit und des Rechts" leben können. Konkret wurden hierzu diverse Rahmenbeschlüsse erarbeitet. Rahmenschlüsse nach Art. 34 II b des EU-Vertrages sind das Mittel der Wahl im Bereich der der Polizeilichen und Justiziellen Zusammenarbeit in 
Strafsachen (PJZS) als einer der drei Säulen, auf denen die EU derzeit (noch) ruht. Prospektiv sollen sie auch zu einer Harmonisierung der nationalen Strafrechtssysteme führen, bislang basieren sie aber vor allem auf dem Prinzip der gegenseitigen Anerkennung von justiziellen Entscheidungen. Sie geben den Rahmen vor, die nationalen Gesetzgeber müssen jedoch binnen drei Jahren nach Inkrafttreten Umsetzungsgesetze schaffen. Das bekannteste Beispiel ist bislang der sog. europäische Haftbefehl, verabschiedet wurden 2008 u. a. ein Rahmenbeschluss zur erleichterten Überstellung von Strafgefangenen zur Vollstreckung ihrer Freiheitsstrafe ${ }^{42}$ und ein Rahmenbeschluss zur grenzüberschreitenden Vollstreckung von Bewährungsstrafen. ${ }^{43}$

Erarbeitet wurde außerdem von der Kommission der Europäischen Union die Vorlage eines Rahmenbeschlusses zur gegenseitigen Anerkennung von Überwachungsmaßnahmen im Ermittlungsverfahren. Zu diesem Zwecke gab es mehrere Expertentreffen, ein sog. „Grünbuch“44 und schließlich den Entwurf eines Rahmenbeschlusses, der zunächst kurz „Europäische Überwachungsanordnung („European Supervision Order, ESO“) hieß und inzwischen offiziell "Council Framework Decision on the application, between Member States of the European Union, of the principle of mutual recognition to decisions on supervision measures as an alternative to provisional detention" getauft ist. ${ }^{45}$ Die Begründung der Kommission zur Notwendigkeit eines solchen Vorschlags fußt zum einen auf einer der zentralen Grundfreiheiten - der Freizügigkeit aller Bürger innerhalb der EU - beruft sich aber ebenso auf die EMRK und hier vor allem auf die Unschuldsvermutung. Die Grundidee ist, dass nach der Entscheidung, statt Untersuchungshaft eine alternative Maßnahme zur Absicherung des Verfahrens anzuordnen, diese Entscheidung zusammen mit einem Formblatt (das dem Rahmenbeschluss anhängt) an den Vollstreckungsstaat gesandt wird, vorausgesetzt, die beschuldigte Person hat dem zugestimmt (Art. 9 des Rahmenbeschlusses). Innerhalb einer bestimmten Frist muss der Ausführungsstaat diese Entscheidung anerkennen bzw. ablehnen (nur bestimmte Ablehnungsgründe sind zulässig) und die Überwachung der Maßnahme (gemeint sind vor allem Meldeauflagen, Aufenthaltsverbote, Ausreiseverbote etc.) übernehmen.

Die Rahmenbeschlüsse sind also vor allem dazu da, Fälle mit grenzüberschreitendem Charakter zu erfassen, was zu der vorweg zu erörternden Frage führt, ob im Bereich der Untersuchungshaft hier allgemein und aus deutscher Sicht überhaupt Bedarf besteht. Das wurde von der EU-Kommission behauptet, ${ }^{46}$ von Kritikern bestritten ${ }^{47}$ beides auf recht unsicherer Tatsachengrundlage, weil in Bezug auf die Anzahl von EUAusländern (grundsätzlich nur sie wären erfasst) in Untersuchungshaftanstalten wenig Daten vorliegen. Unbestritten ist zunächst, dass in den letzten Jahrzehnten der Anteil der Ausländer in europäischen Gefängnissen erheblich angestiegen ist und dass dies insbesondere für Untersuchungsgefangene gilt: Der Anteil liegt im Österreich bei knapp $60 \%$, in Belgien, Spanien über 50\%, in Deutschland, Griechenland und Italien deutlich über $40 \% .{ }^{48}$ Andererseits haben die osteuropäischen EU-Mitgliedsstaaten traditionell sehr wenige Ausländer in ihren Gefängnissen. Hier liegen die Anteile in der Untersuchungshaft zwar regelmäßig auch etwas höher als bezogen auf die Gesamtzahl der Gefangenen, dennoch in der Regel nur zwischen $1 \%$ (Rumänien) und $4 \%$ (Slowakei) - lediglich für Slowenien weist die Statistik mehr als $17 \%$ aus. ${ }^{49}$

In den meisten Staaten ist jedoch nicht ohne weiteres herauszufinden, woher diese Ausländer kommen, weil die Nationalität zumindest von den regulären Statistiken oft nicht erfasst wird. Dies ist auch in Deutschland der Fall. Um genauer ermessen zu können, inwieweit eine verstärkte europäische Zusammenarbeit bei der Überwachung von Maßnahmen, die eine Außervollzugsetzung des Haftbefehls ermöglichen, überhaupt Auswirkungen auf Haftzahlen haben könnte, wurden in einer schriftlichen Umfrage bei den Länderjustizministerien Daten zur Herkunft der Untersuchungsgefangenen erbeten (vgl. Tabelle unten). ${ }^{50}$ Bekannt war aus der internationalen (Europarat, s. o.) und der deutschen (Strafvollzugs-) Statistik, dass ausländische Gefangenen im Verhältnis zu ihrem Bevölkerungsanteil aber auch im Verhältnis zu ihrem Anteil an Tatverdächtigen in den Gefängnissen überrepräsentiert ${ }^{51}$ sind - die Europaratsstatistiken geben ihren Gesamtanteil mit ca. 26\% an, die Strafvollzugsstatistik, die nur die Staatsangehörigkeit der Strafgefangenen ausweist, deren Anteil mit 22\% (2007). Dadurch wird einerseits wieder belegt, dass die Überrepräsentation hauptsächlich auf die Untersuchungshaft zurückzuführen ist - ein Punkt, der in der Tat für eine verstärkte Suche nach grenzüberschreitenden Lösungsmöglichkeiten spricht. ${ }^{52}$ Damit ist andererseits aber noch nicht die Frage beantwortet, ob diese innerhalb der EU zu suchen sind. In Deutschland sind erwartungsgemäß die Ausländeranteile in den Bundesländern ganz unterschiedlich - zwischen $10 \%$ in Mecklenburg-Vorpommern und $57 \%$ in Hamburg. Auch diese Daten reflektieren länderspezifisch die demographische Situation und die oben beschriebene Überrepräsentation. Angehörige andere EU-Staaten machen auf die Gesamtzahl aller Untersuchungsgefangenen im Schnitt ca. 15\% aus, je nach Bundesland zwischen 5\% (in Sachsen und SachsenAnhalt) und 22\% in Hessen. Auch wenn die Stichtagsdaten unterschiedlich sind, kann man schätzen, dass im Frühling/Sommer 2008 ständig etwa 1800 EU-Bürger in deutscher Untersuchungshaft saßen. Wenn man bedenkt, dass - auf alle Untersuchungsgefangene bezogen - ca. 93\% ${ }^{53}$ aller Gefangenen zumindest auch wegen Fluchtgefahr inhaftiert waren und dies für Ausländer einerseits in noch höherem Maße zutreffen, gleichzeitig die Fluchtgefahr bei Ihnen aber in der Regel vor allem durch den auswärtigen Wohnsitz gerechtfertigt sein dürfte, ergibt sich hieraus grundsätzlich schon ein Potential für Haftentlastungen innerhalb der EU.

Für die andere Seite der Medaille - wie viele Deutsche saßen im (EU-) Ausland in Haft, d. h. wie viele wären von einer verbesserten grenzüberschreitenden Zusammenarbeit betroffen - gibt es keine zuverlässigen Daten. ${ }^{54}$ Das ist zwar in vielen europäischen Staaten so, ${ }^{55}$ gibt aber Anlass zu einer grundsätzlichen Bemerkung: Inhaftierung im Ausland ist in Deutschland vorwiegend ein privates Problem, bei Bedarf allenfalls gemildert durch konsularische Hilfe. Die Medien nehmen sich zwar gelegentlich eines spektakulären Falles an - sei es die Inhaftierung eines Hochseeanglers in Iran wegen illegalen Grenzübertritts, Cannabis-Schmuggel einer jungen Studentin bei drohender Todesstrafe in Singapur oder die lange Untersuchungshaft des 17-jährigen deutschen Urlaubers in der Türkei wegen sexueller Belästigung einer Minderjährigen. Trotz der beachtlichen Aufmerksamkeit gerade für den letzten Fall und trotz seines innereuropäischen Bezugs ist der Themenkomplex aber soweit ersichtlich nichts, was die Politik besonders beschäftigen würde. Dafür spricht die lapidare Bemerkung des Bundesrates ${ }^{56}$ zum Vorschlag des Rahmenbeschlusses zur Europäischen Überwachungsanordnung, für die deutschen Länderpolizeien gäbe es einen erheblichen Mehraufwand, wenn sie ausländische Anordnungen überwachen müssten. Nach der Intention ersparen sie immerhin 
einem deutschen Staatsbürger Untersuchungshaft, doch das scheint den Aufwand nicht zu rechtfertigen.

Dies ist in anderen EU-Staaten teilweise anders: In Großbritannien berichten nicht nur die Medien regelmäßig über Fälle von inhaftierten Briten im Ausland, es gibt auch starke Nichtregierungorganisationen wie "Fair Trials Abroad" 57 und "Prisoners Abroad" 58 für die Betreuung britischer Gefangener im Ausland, ähnliche Organisationen gibt es in anderen europäischen Staaten. ${ }^{59}$ Die schwedische Regierung schließlich zeigte besonderes Interesse für das Thema der Alternativen zur Untersuchungshaft bzw. auch für die Durchsetzung bestimmter Mindeststandards nachdem ein 21-jähriger Schwede drei Jahre in griechischer Untersuchungshaft verbrachte, bevor er schließlich seine Haftstrafe antreten (und offenbar zu Hause verbüßen) konnte. Diverse EU-Parlamentarier griffen diesen und entsprechende Fälle auf und diskutierten sie mit dem Tenor, dass es innerhalb des propagierten einheitlichen „Raumes der Freiheit, der Sicherheit und des Rechts “ doch nicht angehen könne, dass solche Fälle nicht besser zu lösen sind. ${ }^{60}$ Die Initiative der EU-Kommission erhielt daher von verschiedenen Seiten durchaus Unterstützung.

Ohne hier auf Einzelheiten des geplanten Rahmenbeschlusses eingehen zu können, sieht es derzeit so aus, als hätte er mit besonders vielen Widerständen zu kämpfen. Dafür spricht, dass, obwohl er gegenüber dem ersten Entwurf grundlegend umgestaltet worden ist und eine politische Einigung Ende 2008 erzielt war, noch immer keine Übersetzungen in alle Sprachen vorliegen, allerdings ist er am 23.10.2009 beim Europäischen Ratstreffen in Luxemburg nun verbindlich verabschiedet worden. Auch von deutschen Verbänden kam - obwohl die Grundintention stets begrüßt wurde - am Entwurf überwiegend Kritik. ${ }^{61}$ Neben der grundsätzlichen Kritik an den Rahmenbeschlüssen, die den Wegfall der Überprüfung der beiderseitigen Strafbarkeit betreffen und der bereits genannten Furcht vor personellen Belastungen beim Vollzug fremder Überwachungsanordnungen, sowie einem grundsätzlichen Misstrauen in die Leistungsfähigkeit insbesondere der Justizsysteme der neuen Mitgliedsländer, richtet sie sich vor allem gegen eine vermutete Ausweitung von Überwachung. So sind nach dem Rahmenbeschluss auch Überwachungsan- ordnungen möglich, wenn nach dem Recht des betroffenen Staates nicht von Anfang an Untersuchungshaft angeordnet werden könnte (Erwägungsgrund 4). Dies liegt an den unterschiedlichen Konzepten von Untersuchungshaft bzw. ihren Alternativen in Europa: Während etwa in Deutschland Alternativen nur im Rahmen einer Außervollzugsetzung der Untersuchungshaft nach ( $\$ 116$ StPO) möglich sind, besteht in $\mathrm{z}$. B. in Polen von vornherein eine Auswahl verschiedener Maßnahmen zur Absicherung des Prozesses (die allerdings von unterschiedlichen Voraussetzungen abhängig sind) und z. B. in England/Wales grundsätzlich eine Vermutung für ein Ausreichen von Auflagen („, bail“ im weitesten Sinne). ${ }^{62}$ Soll Untersuchungshaft (weil Auflagen/Alternativen bei einem Ausländer eben vielleicht nicht ausreichen) vermieden werden, müssten solche Auflagen auch im Ausland überprüfbar sein.

In jedem Fall erscheint aber Art. 2 (2) S. 2 des Rahmenbeschlusses entscheidend zu sein, nach dem stets das Recht des anordnenden Staates maßgeblich ist, in Deutschland also $\ 116$ StPO. Art. 1 erklärt außerdem ausdrücklich, dass eine echte Alternative zu Haft vorliegen muss. Misstrauisch

\section{Ausländische Untersuchungsgefangene nach Herkunft}

\begin{tabular}{|c|c|c|c|c|c|}
\hline \multirow[t]{2}{*}{ Bundesland } & \multirow{2}{*}{$\begin{array}{c}\text { Untersuchungs- } \\
\text { gefangene }\end{array}$} & \multicolumn{2}{|c|}{$\begin{array}{l}\text { ausländische Untersuchungs- } \\
\text { gefangene }\end{array}$} & \multicolumn{2}{|c|}{$\begin{array}{c}\text { ausländische Untersuchungsgefangene } \\
\text { aus anderen EU-Staaten }\end{array}$} \\
\hline & & absolute Zahl & $\begin{array}{c}\text { Anteil } \\
\text { (von Spalte 2) }\end{array}$ & absolute Zahl & $\begin{array}{c}\text { Anteil } \\
\text { (von Spalte 2) }\end{array}$ \\
\hline Baden-Württemberg & 1600 & 763 & $48 \%$ & 279 & $17 \%$ \\
\hline Bayern & 2537 & 1095 & $43 \%$ & 474 & $19 \%$ \\
\hline Berlin & 739 & 408 & $55 \%$ & 124 & $17 \%$ \\
\hline Brandenburg & 220 & 50 & $23 \%$ & 31 & $14 \%$ \\
\hline Bremen & 97 & 53 & $56 \%$ & 8 & $8 \%$ \\
\hline Hamburg & 359 & 205 & $57 \%$ & 54 & $15 \%$ \\
\hline Hessen & 950 & 523 & $55 \%$ & 207 & $22 \%$ \\
\hline Mecklenburg-Vorpommern & 215 & 21 & $10 \%$ & 14 & $7 \%$ \\
\hline Nordrhein-Westfalen & 2843 & 1125 & $40 \%$ & 379 & $13 \%$ \\
\hline Niedersachsen & 917 & 322 & $35 \%$ & 90 & $10 \%$ \\
\hline Rheinland Pfalz & 357 & 137 & $38 \%$ & 36 & $10 \%$ \\
\hline Saarland & 136 & 38 & $28 \%$ & 18 & $13 \%$ \\
\hline Sachsen & 491 & 136 & $27 \%$ & 64 & $13 \%$ \\
\hline Sachsen-Anhalt & 202 & 34 & $17 \%$ & 10 & $5 \%$ \\
\hline Schleswig-Holstein & 203 & 69 & $34 \%$ & 22 & $11 \%$ \\
\hline Thüringen & 220 & 26 & $12 \%$ & 9 & $5 \%$ \\
\hline Total (16 Bundesländer) & 12.086 & 5005 & $41,4 \%$ & 1819 & $15,1 \%$ \\
\hline
\end{tabular}

Quelle: eigene Berechnungen nach Angaben der Länderjustizministerien, vgl. Fußnote 50. 
macht allerdings die Formulierung in Art 2 (2) S. 1, nach der der Rahmenbeschluss kein „Recht“ eines Beschuldigten im Strafverfahren auf eine alternative Maßnahme „verleiht“. Will der Beschluss erreichen, was er verspricht, wäre doch wohl im Sinne einer Ermessensreduzierung auf Null davon auszugehen, dass ein ausländischer Beschuldigter sehr wohl das Recht auf ein milderes Mittel (nämlich nicht-stationäre Überwachung im Ausland) hat, außerdem dürfte angesichts der Existenz einer umgesetzten Überwachungsanordnung die Begründung von Fluchtgefahr mit einer Adresse im EUAusland schwerer fallen als bisher. Es bleibt also abzuwarten, wie der nationale, insbesondere der deutsche, Gesetzgeber ihn umsetzt. Es sollte in diesem Zusammenhang im übrigen darauf hingewiesen werden, dass das deutsche Recht mit $\mathbb{1 2 7 a}$ und \132 StPO durchaus Möglichkeiten kennt, das Strafverfahren gegen ausländische Beschuldigte bei Bagatelltaten durch Sicherheitsleistungen abzusichern, im Falle des \$127a StPO auch dann, wenn der Erlass eines Haftbefehls nicht grundsätzlich ausgeschlossen, wegen der mangelnden Schwere der Tat aber unverhältnismäßig wäre. Der Anwendungsbereich dieser Vorschrift wird möglicherweise (Daten liegen, soweit ersichtlich, nicht vor) noch nicht ausreichend ausgeschöpft und hier könnte eine Europäischen Überwachungsanordnung in der Tat zu einer Art net-widening-effect führen.

Als letzter Punkt bleibt noch zu erwähnen, dass die EU-Kommission mit Rückendeckung insbesondere die schwedische Ratspräsidentschaft noch immer den Gedanken verfolgt, Mindeststandards für das strafrechtliche Ermittlungsverfahren in Europa und vor allem Mindeststandards für die Untersuchungshaft als Rahmenbeschluss verbindlich festzulegen. ${ }^{63}$ Die Diskussionen hierüber zeigen aber, dass sowohl auf EUEbene als auch auf nationaler Ebene Experten sehr unterschiedlicher Auffassung darüber sind, ob weitere europäische Standards möglich und nötig sind. ${ }^{64}$ In Bezug auf den Vollzug der Untersuchungshaft liegen schon jetzt gute Empfehlungen des Europarates vor, weniger hoch sind allerdings die Standards aus der Europaratsempfehlung, die auch die Anordnung und Überprüfung der U-Haft betreffen (Empfehlung (2006) 13). Die Diskussionen zeigen, dass ein Zurückfallen hinter diese Standards nicht ausgeschlossen (sondern eher wahrscheinlich) wäre, wenn es um einheitliche rechtverbindliche Vorgaben für die Mitgliedstaaten geht. Das jedoch wäre aber der Glaubwürdigkeit der EU und ihrer Mitgliedstaaten im Hinblick auf Menschenrechtsfragen überaus abträglich.

Die Verfasserin ist wissenschaftliche Assistentin beim Mitheransgeber Frieder Dünkel an der Universität Greifswald.

\section{Literatur:}

Aebi, M.F., Delgrande, N. (2008): Council of Europe Annual Penal Statistics SPACE I Survey 2006. Lausanne. Online erhältlich: http:// www.coe.int/t/e/legal affairs/legal_co-operation/prisons_and_alternatives/ (zuletzt abgerufen 2.10.2009).

Busse, U. (2008): Frühe Strafverteidigung und Untersuchungshaft. Göttingen.

CPT (2007): Report to the German Government on the visit to Germany carried out by the European Committee for the Prevention of Tor ture and Inhuman or Degrading Treatment or Punishment (CPT) from 20 to 23 December 2005. Strasbourg: Council of Europe. www. cpt.coe.int (zuletzt abgerufen 2.10.2009).

Dünkel, F. (2008): Jugendstrafrecht im europäischen Vergleich im Licht aktueller Empfehlungen des Europarats, Neue Kriminalpolitik 20, S. 102-114.

Dünkel, F., Drenkhahn, K. Morgenstern, C. (Hrsg.) (2008): Humanisierung des Strafvollzugs - Konzepte und Praxismodelle. Mönchengladbach.

Dünkel, F., Gensing, A., Morgenstern, C. (2007): Germany. In: van Kalmthout, A, Hofstee-van der Meulen F., Dünkel F (Hrsg.): Foreigners in European Prisons. Nijmegen, S. 341-359.

Dünkel, F., Pruin, I (2009): "Summary Analysis of the national replies to the questionnaire related to the treatment of juvenile offenders" (European Committee on Crime Problems/ Council for Penological Co-operation; PC-CP (2009)04prov. http://www.coe.int/t/e/legal affairs/legal_co-operation/prisons_and_alternatives/ (zuletzt abgerufen 2.10.2009).

Dünkel, F., Walter, J. (2005): Young foreigners and members of ethnic minorities in German youth prisons. In: Queloz, N. u. a. (Hrsg.) Youth Crime and Juvenile Justice. The challenge of migration and ethnic diversity. Bern, S. 517-540.

Feest, J., Köhne, M. (2008): Eine gesetzliche Grundlage für den Vollzug der Untersuchungshaft. Online erhältlich: www.strafvollzugsarchiv.de (zuletzt abgerufen am 20. August 2009).

Jehle, J.-M. (1995): Entwicklung der Untersuchungshaft bei Jugendlichen und Heranwachsenden vor und nach der Wiedervereinigung. Bonn.

Kazele, N. (2008): Untersuchungshaft. Ein verfassungsrechtlicher Leitfaden für die Praxis. Baden-Baden.

Morgenstern, C. (2008): Strafvollstreckung im Heimatstaat - der geplante EU-Rahmenbeschluss zur transnationalen Vollstreckung von Freiheitsstrafe. Zeitschrift für internationale Strafrechtsdogmatik (ZIS), S. 76-83.

Morgenstern, C. (2009a): Pre-trial/remand detention in Europe: facts and figures and the need for common minimum standards. ERA-Forum: Vol. 9, Issue 4, S. 527-542. http://www. springerlink.com/openurl.asp ? genre $=$ article \&id=doi:10.1007/s12027-008-0096-x.

Morgenstern, C. (2009b): Germany. In: van Kalmthout, A., Knapen, M., Morgenstern, C. (Hrsg.): Pre-trial Detention in Europe. Nijmegen. (im Erscheinen)
Paeffgen, H.-U. (1986): Vorüberlegungen zu einer Dogmatik des U-Haftrechts. Köln.

Schöch, H., Jehle, J.-M. (Hrsg.) (2004): Angewandte Kriminologie zwischen Freiheit und Sicherheit. Mönchengladbach.

Seifert, H.-J. (2008): Wohngruppenvollzug für Untersuchungsgefangene in der JVA Moabit III. In: Dünkel, F., Drenkhahn, K., Morgenstern, C. (Hrsg.) (2008) Humanisierung des Strafvollzugs - Konzepte und Praxismodelle. Mönchengladbach, S. 73-80.

van Kalmthout, A. u. a. (2009): Introductory Summary. In: van Kalmthout, A., Knapen, M., Morgenstern, C. (Hrsg.) (2009): Pre-trial Detention in Europe. Nijmegen. (im Erscheinen)

van Kalmthout, A., Hofstee-van der Meulen, F., Dünkel, F. (Hrsg.) Foreigners in European Prisons. Nijmegen.

van Kalmthout, A., Knapen, M., Morgenstern, C. (Hrsg.) (2009): Pre-trial Detention in Europe. Nijmegen. (im Erscheinen)

Weigend, T., Salditt F. (2007) The investigative stage of the criminal process in Germany. In: Cape E. u. a. Suspects in Europe. Brüssel, S.78-99.

\section{Fußnoten:}

1 Z. B. Paeffgen 1986; einen Überblick über die relevante höchstrichterliche Rechtsprechung gibt Kazele 2008. Vgl. hierzu auch den Beitrag von Ostendorf in diesem Heft.

2 Dünkel/Drenkhahn/Morgenstern 2009, zum Berliner Projekt Seifert 2009.

3 Vgl. zu den Entwürfen den Beitrag von Höflich in diesem Heft.

4 Entwurf eines Gesetzes zur Änderung des Untersuchungshaftrechts, BT-Drs. 16/11644 vom 21.1.2009, Beschlussempfehlung des Rechtsausschusses, BT-Drs. 16/13097 vom 20.05.2009.

5 Feest/Köhne 2008.

6 Die Entwürfe von Berlin, Brandenburg, Bremen, Hamburg, Hessen, Mecklenburg-Vorpommern, Rheinland-Pfalz, Saarland, Sachsen, SachenAnhalt, Schleswig-Holstein und Thüringen basieren auf dem Entwurf einer gemeinsamen Arbeitsgruppe.

7 Vgl. hierzu Kazele 2008 (siehe auch die Rezension in diesem Heft).

8 Görgülü-Entscheidung des Bundesverfassungsgerichts von 2004, BVERfGE 111,307.

9 Weigend/Salditt 2007, S. 80.

10 Mooren vs. Deutschland, No. 11364/03, Entscheidung vom 13. Dezember 2007 (Kammerentscheidung) und Entscheidung vom 9. Juli 2009 (große Kammer). Eine große Kammerentscheidung war wegen eines Rechtmittels des Betroffenen bezüglich seines Antrags, dass die Haftfortdauer an sich rechtswidrig gewesen sei, nötig geworden.

11 Erdem vs. Germany, No. 38321/97, 5 July 2001; Lietzow vs. Germany, No. 24479/94, 13 February 2001; Garcia Alva vs. Germany, No. 23541/94, 13 February 2001.

12 Expertenanhörung im Rechtsausschuss des Bundestags vom 22.4.2009; Plenardebatte der ersten Lesung des Entwurfs vom 12. Februar 2009, PlProt 16/205, S. 22196 ff.

13 Paeffgen 1986, Busse 2008, verschiedene Beiträge in Schöch/Jeble 2004.

14 Erdem c. Allemagne, No. 38321/97, 5 Juillet 2001 (U-Haft 5 Jahre und 11 Monate); Cevizovic vs. Germany, No. 49746/99, 29 October 2004 (U Haft 4 Jahre und 9 Monate) und Dzelili vs. Germany, No. 65745/01, 10 February 2006 (U-Haft 5 Jahre und 4 Monate); in allen drei Fällen wurde ein Verstoß gg. Art. 5 III EMRK angenommen.

15 In Westdeutschland betrug der höchste Stichtagsstand im März 1994 fast 19.400 Untersuchungshäftlinge, im März 2003 waren es ca. 14.600, im März 2008 weniger als 11.000. In 
den östlichen Bundesländern gingen die Zahlen von mehr als 2300 im März 2003 auf 1414 im März 2008 zurück (vgl. Morgenstern 2009b, Figure $4 \mathrm{a}$ und $4 \mathrm{~b})$.

16 Morgenstern 2009a mit eigenen Berechnungen anhand von Daten aus der Strafverfolgungsstatistik.

17 z. B. Montag in der 1. Beratung des Gesetzentwurfs vom 12.2.2009, König in der Stellungnahme für den Rechtsausschuss (vgl. oben Fußnote 12 ).

18 Statt aller: Paeffgen 1987, S. 57; Literaturzusammenstellung bei Kazele 2008, S. 120.

19 Chraidi vs. Germany, No. 65655/01 of 26 January 2007. Weitere Fälle Art. 5 III EMRK betreffend sind seitdem verhandelt, aber als unzulässig erklärt worden.

20 van Kalmthout/Knapen/Morgenstern 2009

21 van Kalmthout u. a. (2009), Introductory summary, unter 6.

22 CPT 2007, S. $15 \mathrm{ff}$.

23 Vgl. hierzu die geäußerten Zweifel des Justizpraktikers (Ermittlungsrichters) in der Stellungnahme von Buckow (vgl. Fußnote 12).

24 Seit 1990 führte das CPT mehr als 270 Besuche durch (davon fünf in Deutschland), über 220 Berichte sind, in nahezu allen Fällen mit dem Einverständnis der jeweiligen Regierungen, veröffentlicht. Die sog. CPT-Standards beschreiben die relevanten Anliegen, die das CPT bei den Besuchen verfolgt, so dass die Regierungen und Justizverwaltungen eine klare Vorstellung davon gewinnen können, was von ihnen erwartet wird (d. h. wie Inhaftierte nach den Vorstellungen des CPT zu behandeln sind); www.cpt. coe.int/en/docsstandards.htm.

25 Auch der baden-württembergische Entwurf sieht in $\$ 34$ solch ein Arbeitsangebot vor. Verfassungs- und konventionswidrig ist aber der dort auch zu findende Satz „Untersuchungsgefangene können auch zu Hilfstätigkeiten in der Justizvollzugsanstalt herangezogen werden.“, denn nach dem Wortlaut fordert er keine $\mathrm{Zu}$ stimmung der Untersuchungsgefangenen, ein klarer Verstoß gegen die Usnchuldvermutung

26 CPT 2007, S. $10 \mathrm{ff}$.

27 Zitat aus dem Bericht: „Üblicherweise erfolgte die Fixierung an Arm(en), Bein(en) und/oder Rumpf von Personen, die auf einem Bett/einer Matratze auf dem Rücken lagen, wobei die Fixierung durch Gurte oder Handschellen aus Metall erfolgte."

28 Alle Empfehlungen des Europarates sind in der auf englisch und französisch abrufbar unter http://www.coe.int/t/cm/documentIndex en.asp, unter „Recommendations of the Committee of Ministers to member states “.

29 Entscheidung des Bundesverfassungsgerichts vom 31.5.2006, Rn. 63; NJW 2006, 2093 ff.

30 Meyer-Goßner 2008, \$113 Rdnr. 1.

31 In der Strafverfolgungstatistik 2007 war keine Verurteilung mit vorangegangener Untersuchungshaft wegen eines Deliktes zu finden, das abstrakt keine Androhung der Freiheitsstrafe beinhaltet. Allerdings sind die Strafvorschriften, bei denen abstrakt nur Geldstrafe angedroht ist, auch sehr selten.

32 Vgl. van Kalmthout u. a. (2009), 4.2 („,preconditions for pre-trial detention“). Ausnahmen gibt es, z. B. Griechenland, wo grundsätzlich nur eine Person, die eines Verbrechens („felony“) beschuldigt worden ist, in Untersuchungshaft genommen werden kann (was nahezu alle Fahrlässigkeitstaten ausschließt), oder Finnland, wo eine Freiheitsstrafe von mind. zwei Jahren angedroht sein muss.

33 Nachweise bei Kazele 2008, S. $119 \mathrm{ff}$

34 Recommendation Rec (2006)2 of the Committee of Ministers to member states on the European Prison Rules, abrufbar unter https://wcd. coe.int. Eine von den Justizministerien Deutschlands, Österreichs und der Schweiz autorisierte deutsche Übersetzung ist auch erhältlich (Hrsg. von den Bundesministerien der Justiz Berlin, Wien und Bern 2007).

35 Klargestellt z. B. in BVerfG NStZ 2008, S. 521.

36 BVerfGE vom 23. Oktober 2006 (2 BvR 1797/06).

37 Van Kalmthout/Knapen/Morgenstern 2009 in den jeweiligen Länderkapiteln und Introductory summary, 7.4 .

38 Information über die Internetseite der englischen Gefängnisbehörde (Her Majesty‘s Prison Service), http://www.hmprisonservice.gov. uk/adviceandsupport/keepingintouch/visitorsguide/.

39 Vgl. hierzu z. B. Dünkel 2008 und mit mehr Details zur Umsetzung von Standards in europäischen Staaten Dünkel/Pruin 2009.

40 European Council, EU Presidency Conclusions, 4-5 November 2004, Annex I: The Hague Programme: Strengthening Freedom, Security and Justice in the European Union, Brussels, 13 December 2004, Council Document 16054/04.

41 KOM (2009) 262/4: Mitteilung der Kommission an das Europäische Parlament und den Rat, deren zweite Überschrift lapidar „Mehr Freiheit und mehr Sicherheit" lautet.

42 Rahmenbeschluss des Rates 2008/909/JHA vom 27. November 2008 (on the application of the principle of mutual recognition to judgments in criminal matters imposing custodial sentences or measures involving deprivation of liberty for the purpose of their enforcement in the European Union); vgl. hierzu Morgenstern 2008.

43 Rahmenbeschluss des Rates 2008/947/JHA vom 27 November 2008 (on the application of the principle of mutual recognition to judgments and probation decisions with a view to the supervision of probation).

$44 \mathrm{Vgl}$. im Einzelnen das Kommissionsdokument $\operatorname{KOM}(2004) 562$ endg.

45 Council document 17002/08 COPEN 249, Brussels, 12 December 2008.

46 Kommissionsdokument KOM (2006) 468 endg.; Ratsdokument 12367/06.

47 In einer Empfehlung des Bundesrates heißt es, der Anwendungsbereich des geplanten Rahmenbeschluss sei klein, weil „die Grundannahme der Kommission, gegen gebietsfremde Beschuldigte werde übermäßig Untersuchungshaft vollstreckt, so nicht zutrifft.", BR-Drs. 654/1/06, Nr. 6.

48 Vgl. ausführlicher Morgenstern 2009a, Tab. 1, basierend auf den Daten des Europarates für 2006, Aebi/Delgrande 2008. Die inzwischen auch bis 2007 vorliegenden Daten ergeben kein anderes Bild.

49 Estland mit mehr als 46\% ist ein Sonderfall, weil hier alle Inländer, die (noch) keinen estnischen Pass besitzen und in der Regel russischer Nationalität sind, in die Statistik einfließen.

50 Korrespondenz der Verfasserin mit den Länderjustizministerien bzw. mit den Anstalten (Hamburg) selbst; Stichtags-Daten für den 31.März 2008 außer Hamburg (24. Juli 2008); Schleswig-Holstein, Mecklenburg-Vorpommern und Bremen (31. Juli 2008), Sachsen-Anhalt und Rheinland-Pfalz (31. August 2008).

51 Jehle 1995; für junge Gefangene vgl. Dünkel/ Walter 2005.

52 Und gegen die Auffassung des Bundesrates, vgl. oben Fußnote 47.

53 Eigene Berechnungen nach der Strafverfolgungsstatistik für 2007 (nur für Untersuchungshaft bei Erwachsenen).

54 Dünkel/Gensing/Morgenstern 2007, S. 387 ff.

55 van Kalmthout/Hofstee-van der Meulen/Dünkel 2007, S. $70 \mathrm{ff}$.

56 BR-Drs. 654/1/06, Nr. 6.

57 http://www.fairtrials.net/index.php/cases/.

58 www.prisonersabroad.org.uk.
59 Sie sind z. B. zusammengeschlossen in einer Expertengruppe der Europäischen Bewährungshilfekonferenz (CEP), CEP Expert Group on Foreign Nationals, www.cepprobation.org. Eine deutsche Mitgliedsorganisation gibt es nicht.

60 Die Fälle wurden in einer Debatte des Europäischen Parlamentes vorgetragen und diskutiert (European Parliament: Debate on the future of the area of freedom, security and justice, 13 October 2004, Brussels; http://www.europarl. europa.eu).

61 Z. B. Antrag der grünen Fraktion im Bundestag vom 6.5.2009, BT-Drs. 16/12856; BR-Drs. BR-Drs. 654/1/06 (vgl. auch oben); kleine Anfrage der FDP-Fraktion BT-Drs. 16/11293; Stellungnahme der Bundesrechtsanwaltskammer (BRAK-Stellungnahme Nr. 38/2006, www. brak.de); Stellungnahme der Fachgruppe Strafrecht der Neue Richtervereinigung (http://www. nrv-net.de/main.php?id=170\&fg id=6). Zu beachten ist, dass sich die Stellungnahmen überwiegend auf das Ursprungsdokument (KOM (2006) 468 endg.) beziehen.

62 van Kalmthout u. a. 2009, 7.3 Alternatives to pre-trial detention; vgl. auch den Landesbericht "United Kingdom“ in van Kalmthout/Knapen/ Morgenstern (Hrsg.) 2009.

$63 \mathrm{Vgl}$. hierzu das Stockholm-Programm (oben Fußnote 41), S. 19.

64 „Expert meeting held in Brussels in June 2006", das Protokoll des Treffens ist erhältlich unter http://ec.europa.eu/justice_home/news/ events/expert_pre_trial/meeting_report_en.pdf Ein weiteres Treffen fand mit ebenso uneinheitlichem Ergebnis am 9. Juni 2009 statt. 